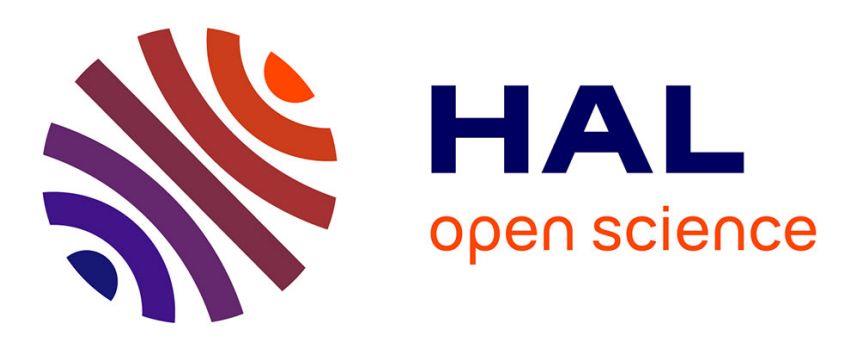

\title{
A Polynomial Approach for Maxima Extraction and Its Application to Tractography in HARDI
}

\author{
Aurobrata Ghosh, Demian Wassermann, Rachid Deriche
}

\section{To cite this version:}

Aurobrata Ghosh, Demian Wassermann, Rachid Deriche. A Polynomial Approach for Maxima Extraction and Its Application to Tractography in HARDI. Information Processing in Medical Imaging, Jul 2011, Irsee, Germany. pp.723-734, 10.1007/978-3-642-22092-0_59 . inria-00610195

\section{HAL Id: inria-00610195 \\ https://hal.inria.fr/inria-00610195}

Submitted on 21 Jul 2011

HAL is a multi-disciplinary open access archive for the deposit and dissemination of scientific research documents, whether they are published or not. The documents may come from teaching and research institutions in France or abroad, or from public or private research centers.
L'archive ouverte pluridisciplinaire HAL, est destinée au dépôt et à la diffusion de documents scientifiques de niveau recherche, publiés ou non, émanant des établissements d'enseignement et de recherche français ou étrangers, des laboratoires publics ou privés. 


\title{
A Polynomial Approach for Maxima Extraction and its Application to Tractography in HARDI *
}

\author{
Aurobrata Ghosh ${ }^{1}$, Demian Wassermann ${ }^{2}$, and Rachid Deriche ${ }^{1}$ \\ 1 Athéna Project Team, INRIA Sophia Antipolis-Méditerranée, France, \\ \{Aurobrata.Ghosh, Rachid.Deriche\}@inria.fr, \\ 2 Department of Radiology, Harvard Medical School \& Brigham and Women's \\ Hospital, Boston, USA
}

\begin{abstract}
A number of non-parametrically represented High Angular Resolution Diffusion Imaging (HARDI) spherical diffusion functions have been proposed to infer more and more accurately the heterogeneous and complex tissue microarchitecture of the cerebral white-matter. These spherical functions overcome the limitation of Diffusion Tensor Imaging (DTI) at discerning crossing, merging and fanning axonal fiber bundle configurations inside a voxel. Tractography graphically reconstructs the axonal connectivity of the cerebral white-matter in vivo and noninvasively, by integrating along the direction indicated by the local geometry of the spherical diffusion functions. Tractography is acutely sensitive to the local geometry and its correct estimation. In this paper we first propose a polynomial approach for analytically bracketing and numerically refining with high precision all the maxima, or fiber directions, of any spherical diffusion function represented non-parametrically. This permits an accurate inference of the fiber layout from the spherical diffusion function. Then we propose an extension of the deterministic Streamline tractography to HARDI diffusion functions that clearly discern fiber crossings. We also extend the Tensorline algorithm to these HARDI functions, to improve on the extended Streamline tractography. We illustrate our proposed methods using the Solid Angle diffusion Orientation Distribution Function (ODF-SA). We present results on multitensor synthetic data, and real in vivo data of the cerebral white-matter that show markedly improved tractography results.
\end{abstract}

\section{Introduction}

Diffusion MRI (dMRI) is a state-of-the-art method for studying the complex micro-architecture of the cerebral white matter in vivo and non-invasively. dMRI is sensitive to and measures the diffusion of water molecules. The complex geometry of the underlying tissue can be inferred by fundamentally assuming that the diffusion of water molecules is relatively less hindered parallel to coherent microstructures, such as axonal fiber bundles, than perpendicular to these structures. Hence the geometry or shape of the reconstructed diffusion function is an

\footnotetext{
* This work was partially supported by the ANR project NucleiPark and the France-
} Parkinson Association. 
indicator of the microarchitecture of the tissue. The orientation information, which indicates axon fiber bundle directions, is of greater importance than the radial part, therefore, naturally the diffusion function is represented as a spherical function. However, it is well known that the current spatial resolution of dMRI at typically $2 \mathrm{~mm}^{3}[9]$ is coarse compared to the true scale of an axon, which is of the order of $1 \mu \mathrm{m}$. Therefore, the spherical diffusion function (SDF) represents at best the average or dominant fiber direction of the underlying tissue locally, and is affected by partial voluming effects.

Diffusion Tensor Imaging (DTI) [4] is the most popular and well-utilized dMRI reconstruction protocol. DTI is known to work well in regions with a single fiber configuration, but to become ambiguous in regions where fiber bundles cross, merge, fan or kiss. Since about a third of dMRI voxels contain such complex fiber configurations [6], SDFs with richer geometries and multiple peaks capable of discerning crossings, have been of central importance in recent dMRI research. Diffusion acquisition protocols have also evolved to overcome this limitation of DTI with High Angular Resolution Diffusion Imaging (HARDI). A number of non-parametrically represented HARDI SDFs have been recently proposed that can indicate fiber crossings. e.g. the Orientation Distribution Function (ODF) [18], the Fiber Orientation Density (FOD) [17], the Persistent Angular Structure (PAS) [2], and the Diffusion Orientation Transform (DOT) [16].

Tractography graphically reconstructs the connectivity of the cerebral whitematter by integrating along the SDF's geometry locally. It is a modern tool that is unique in the sense that it permits an indirect dissection visualization of the brain in vivo and non-invasively [8]. The underpinnings of tractography are also based on the fundamental assumption of dMRI, i.e. that the diffusion of water molecules is hindered to a greater extent perpendicular to coherent fiber bundle structures than parallel to these. Therefore, following the geometry of the local diffusion function and integrating along reveals the continuous dominant structure of the fiber bundle. However, tractography is acutely sensitive to the local geometry, and its correct estimation is crucial.

Deterministic tractography has seen considerable success in researching neurological disorders [9]. Classically it was defined on DTI [5, 21]. However, since DTI is ambiguous in regions with fiber crossings, the trend in recent years has been to extend tractography to complex SDFs that describe fiber directions more accurately $[20,12]$. Probabilistic tractography was proposed to address the reliability of deterministic tractography which remains sensitive to a number of parameters. Probabilistic tractography measures the likelihood of two regions being connected. Given the capabilities of dMRI schemes of date, due to partial voluming, noise, etc., probabilistic tractography provides a more complete statement. However, there exist state-of-the-art probabilistic schemes that rely on deterministic tracking to compute likelihood measures [14]. Therefore, deterministic tractography is an important problem.

In this paper we propose a deterministic tractography scheme based on a polynomial approach for accurately extracting the maxima of any non-parametrically represented SDF. Our paper has two main contributions. First, the polynomial 
maxima extraction we use can analytically bracket all the maxima of any spherical (diffusion) function (SDF). This is neither a heuristic approach, like a finite difference mesh search, nor a local search approach, like optimization. It guarantees that all the maxima are located analytically, and then refined numerically to any degree of precision based on known numerical schemes. This ensures that given a SDF we can accurately quantify its local geometry for tracking, and that no maxima are overlooked. Computationally, we find in our experiments that the tracking time using our approach is comparable to the discrete mesh search proposed in [12]. Our maxima extraction can be considered the limiting case of the mesh search in [12] with a complete mathematical framework like in an optimization approach. Essentially it operates on the continuous SDF and locates all its extrema, i.e. $\nabla(\mathrm{SDF})=\mathbf{0}$, while not being dependent on initialization.

The second contribution of our paper is in our extension of the classical DTI Streamline tractography [5]. We adapt the Streamline tractography to the multiple maxima that can be discerned by complex SDFs in regions with fiber crossings, allowing us to trace through such regions with greater accuracy. We also extend the well known Tensorline tractography [21] to complex SDFs, to smooth out local kinks that can make the fiber tracks unnaturally "wriggly" in plain Streamline tractography due to acquisition noise and partial voluming which make the estimated SDF field spatially irregular. This is important, since kinks can violate the curvature threshold and stop the tracking algorithm.

We illustrate our method using Solid Angle diffusion ODFs (ODF-SA) [1]. The ODF-SA is a non-parametric SDF represented in the Spherical Harmonic (SH) basis and is a good generic SDF for applying our maxima extraction method. We first experiment on synthetic data generated from a multi-tensor model, then on in vivo human cerebral data [3] where we show marked improvements in detecting lateral radiations of the Corpus Callosum using our modified Tensorline tractography.

\section{Materials and Methods}

We first describe in detail our polynomial maxima extraction method. Then we describe the extensions of the Streamline and the Tensorline algorithms to complex SDFs.

Maxima Extraction: A Polynomial Approach The SDF of DTI is the diffusion ellipsoid parametrized by the $3 \times 3$ symmetric tensor. Its geometry can be easily quantified from the eigen-decomposition of the tensor, e.g. the major eigenvector indicates the locally dominant fiber direction. However, quantifying the geometry of more complex SDFs with multiple peaks isn't evident. We propose here a polynomial framework for extracting the maxima of such SDFs.

Our approach can be broken down into three steps. First, we rewrite a nonparametric SDF described in the SH basis, which is the most popular basis used in the dMRI, in an equivalent homogeneous polynomial basis constrained to the sphere. Second, we formulate a constrained polynomial optimization problem for identifying all the stationary points or extrema of the SDF. We then solve it 
using a novel polynomial system solver instead of a local optimization approach. We can do so since the optimization problem can be shown to be a root finding problem for a system of homogeneous polynomials. This approach allows us to analytically bracket all the real roots of the polynomial system without depending on an initial solution. We refine the roots numerically to a high precision to accurately quantify all the extrema of the SDF. Finally in the third step, we categorize the extrema into maxima, minima and saddle-points of the SDF and thus extract all the maxima of the SDF.

The SH basis is an ideal formulation for describing spherical functions, e.g. SDFs with rich geometries, non parametrically, since they form a complex complete orthonormal basis for square integrable functions on the unit sphere. A modified, real, and symmetric SH basis is popularly used in dMRI to describe SDFs [11], since the diffusion function is real and assumed to be symmetric:

$$
Y_{j}(\theta, \phi)= \begin{cases}\sqrt{(2)} \cdot \operatorname{Re}\left(Y_{l}^{|m|}(\theta, \phi)\right) & \text { if } m<0 \\ Y_{l}^{m}(\theta, \phi) & \text { if } m=0 \\ \sqrt{(}(2) \cdot \operatorname{Im}\left(Y_{l}^{m}(\theta, \phi)\right) & \text { if } m>0\end{cases}
$$

where $j=\left(l^{2}+l+2\right) / 2+m, \theta \in[0, \pi], \phi \in[0,2 \pi)$, and $Y_{l}^{m}(\theta, \phi)$ are the SHs.

The space of truncated SHs of rank- $d$ is linearly bijective to the space of homogeneous polynomials (HP) of degree- $d$ constrained to the sphere [10]. If a spherical function is described by a HP: $\mathrm{P}\left(\mathbf{x}=\left[x_{1}, x_{2}, x_{3}\right]^{T}\right)=\sum_{i_{1}+i_{2}+i_{3}=d}$ $A_{i_{1} i_{2} i_{3}} x_{1}^{i_{1}} x_{2}^{i_{2}} x_{3}^{i_{3}}$, where $\left(\|\mathbf{x}\|_{2}=1\right)$ or $x_{i}=x_{i}(\theta, \phi), i=1 . .3$, then the SH coefficients of this spherical function can be computed from the spherical harmonic transform (SHT):

$$
c_{j}=\sum_{i_{1}+i_{2}+i_{3}=d} A_{i_{1} i_{2} i_{3}} \int_{\Omega} x_{1}^{i_{1}}(\theta, \phi) x_{2}^{i_{2}}(\theta, \phi) x_{3}^{i_{3}}(\theta, \phi) \cdot Y_{j}(\theta, \phi) d \Omega .
$$

Since the coefficients $A_{i_{1} i_{2} i_{3}}$ of the HP are outside the integration in the SHT Eq.2 can be rewritten as a linear transformation $\mathbf{C}=\mathbf{M A}$ by reordering the indices $i_{1}, i_{2}, i_{3}$ suitably, where $\mathbf{C}$ are the coefficients in the $\mathrm{SH}$ basis, $\mathbf{A}$ the coefficients in the HP basis, and $\mathbf{M}$ is the transformation matrix. When $\mathbf{C}$ and $\mathbf{A}$ have the same dimension, $\mathbf{M}$ becomes an invertible square matrix, and $\mathbf{A}$ can be computed from $\mathbf{C}$. In other words a SDF described in a truncated SH basis of rank- $d$ can be equivalently rewritten in a constrained HP basis of degree- $d$.

Finding the maxima of the SDF can be formulated as a constrained optimization (maximization) problem: $\max _{\mathbf{x}} \mathrm{P}(\mathbf{x})$ subject to $\|\mathbf{x}\|_{2}^{2}-1=0$. Using Lagrange Multipliers, it can be rewritten as an unconstrained functional: $\mathrm{F}(\mathbf{x}, \lambda)=\mathrm{P}(\mathbf{x})-\lambda\left(\|\mathbf{x}\|_{2}^{2}-1\right)$. From optimization theory, the maxima $\mathbf{x}^{*}$ (and its corresponding $\left.\lambda^{*}\right)$ of the SDF would have to satisfy $\nabla \mathrm{F}\left(\mathbf{x}^{*}, \lambda^{*}\right)=0$ :

$$
\frac{\partial \mathrm{F}\left(\mathbf{x}^{*}, \lambda^{*}\right)}{\partial x_{1}}=\frac{\partial \mathrm{F}\left(\mathbf{x}^{*}, \lambda^{*}\right)}{\partial x_{2}}=\frac{\partial \mathrm{F}\left(\mathbf{x}^{*}, \lambda^{*}\right)}{\partial x_{3}}=\left\|\mathbf{x}^{*}\right\|_{2}^{2}-1=0 .
$$

Eq.3 is a system of HPs, $\left\{\mathrm{Q}_{k}(\mathbf{X})\right\}$, and implies that $\mathbf{X}^{*}=\left(\mathbf{x}^{*}, \lambda^{*}\right)$ would be a root of this system. Since the SDF is a real function, only the real roots of this 
system are of interest. However, $\nabla \mathrm{F}(\tilde{\mathbf{x}}, \tilde{\lambda})=0$ identifies all the stationary points or extrema of the SDF. Therefore, once the real roots of the SDF are identified, they would have to be categorized into the maxima, minima, and saddle-points.

To find the real roots of Eq.3 we use the subdivision solver for polynomial systems proposed in [15]. The solver works as follows. The polynomials are converted from the monomial basis to the Bernstein basis, since the latter has intuitive geometric interpretations:

$$
\begin{aligned}
\mathrm{Q}(\mathbf{X})=\sum_{i_{1}=0}^{d_{1}} \sum_{i_{2}=0}^{d_{2}} \sum_{i_{3}=0}^{d_{3}} \sum_{i_{4}=0}^{d_{4}} B_{i_{1}, i_{2}, i_{3}, i_{4}} & \mathrm{~B}_{d_{1}}^{i_{1}}\left(x_{1} ; a_{1}, b_{1}\right) \mathrm{B}_{d_{2}}^{i_{2}}\left(x_{2} ; a_{2}, b_{2}\right) . \\
& \mathrm{B}_{d_{3}}^{i_{3}}\left(x_{3} ; a_{3}, b_{3}\right) \mathrm{B}_{d_{4}}^{i_{4}}\left(x_{4}=\lambda ; a_{4}, b_{4}\right),
\end{aligned}
$$

where $\mathrm{B}_{d}^{i}(x ; a, b)=\left(\frac{d}{i}\right) \frac{1}{(b-a) d}(x-a)^{i}(b-x)^{d-i}$ form the Bernstein basis on $[a, b]$ along any dimension, and the domain of $\mathrm{Q}(\mathbf{X})$ is $\left[a_{1}, b_{1}\right] \times\left[a_{2}, b_{2}\right] \times\left[a_{3}, b_{3}\right] \times$ $\left[a_{4}, b_{4}\right]$. Since the SDF is defined on the unit sphere, the initial domain of all the $\mathrm{Q}(\mathbf{X})$ 's can be $[-1,1] \times[-1,1] \times[-1,1] \times[-K, K]$, for very large $K$. The coefficients are converted to the Bernstein basis using exact arithmetic to avoid loss of precision. This operation is required once.

Along every dimension, De Casteljau's algorithm efficiently subdivides the Bernstein representation of any $\mathrm{Q}(\mathbf{X})$ into two sub-representations in two subdomains of the initial domain. This allows to subdivide the entire domain into sub-domains efficiently by subdividing along all four dimensions to locate the roots. Moreover, along every dimension Descartes' theorem states that the number of real roots of $q(x)=\sum b_{i} B_{d}^{i}(x ; a, b)$ in $] a, b[$ is bounded by the number of sign changes of $\left\{b_{i}\right\}$ and is equal modulo 2, where $q(x)$ is the projection of $\mathrm{Q}(\mathbf{x})$ along that dimension. This implies an exclusion test that is negative when there are no real roots in the interval and is positive when there are one or more real roots in the interval. Therefore, making it possible to analytically identify and subdivide intervals along every dimension to bracket the real roots. Once a root has been bracketed or isolated any standard numerical one dimensional root-finder of choice can be used to refine the real root with high precision.

From the Bernstein coefficients in Eq.4 it is easy to sandwich the projection $q(x)$ of $\mathrm{Q}(\mathbf{X})$ along any dimension $j$ by $m_{j}\left(q ; x_{j}\right)=\sum_{i_{j}}^{d_{j}} \min _{\left(0 \leq i_{k} \leq d_{k}, k \neq j\right)} B_{i_{1}, i_{2}, i_{3}, i_{4}}$ $\mathrm{B}_{d_{j}}^{i_{j}}\left(x_{j} ; a_{j}, b_{j}\right)$ and by $M_{j}\left(q ; x_{j}\right)=\sum_{i_{j}}^{d_{j}} \max _{\left(0 \leq i_{k} \leq d_{k}, k \neq j\right)} B_{i_{1}, i_{2}, i_{3}, i_{4}} \mathrm{~B}_{d_{j}}^{i_{j}}\left(x_{j} ; a_{j}, b_{j}\right)$, such that the roots of $q\left(x_{j}\right)$ (along dimension $j$ ) are sandwiched by the roots of $m_{j}$ and $M_{j}$ [15]. Thus if $m_{j}$ and $M_{j}$ don't have any roots in an interval $\left[a_{j}, b_{j}\right]$, as indicated by Descartes' theorem applied to $m_{j}$ and $M_{j}$, then $q\left(x_{j}\right)$ has no root in that interval, as implied by the exclusion test. Furthermore, if any of the projections $q_{k}\left(x_{j}\right)$ of the system of polynomials $\mathrm{Q}_{k}(\mathbf{X})$ has no roots in the interval $\left[a_{j}, b_{j}\right]$, then the system $\mathrm{Q}_{k}(\mathbf{X})$ has no real roots in the sub-domain overlapping this interval, and the sub-domain can be discarded.

The powerful exclusion test allows to analytically subdivide and reject subdomains of the initial domain in such a fashion that the rejected sub-domains are guaranteed to not contain any real roots of Eq.3. Once the intervals that were not 
rejected along every dimension are small enough, they are numerically refined to locate the roots along those dimensions in those intervals. However, theoretically, these roots (along given dimensions) are only roots of the projections $q_{k}\left(x_{j}\right)$ and may not be a root $\tilde{\mathbf{X}}$ of $\mathrm{Q}_{k}(\mathbf{X})$. An additionally test would be required to reject solutions to the above subdivision process that may not be real roots of the polynomial system. In practice, however, we have never come across such solutions, but simply testing for $\nabla \mathrm{F}(\tilde{\mathbf{X}})=0$ would provide such a test.

Once all the extrema of the SDF have been quantified precisely, these would have to be categorized into maxima, minima and saddle-points to identify the maxima of the SDF. We use the Bordered Hessian test [13] to do this. In unconstrained multi-dimensional optimization the extremum, $\tilde{\mathbf{x}}$, can be categorized by evaluating the Hessian, $H_{\mathbf{x}} \mathrm{F}(\tilde{\mathbf{x}})$. If $H_{\mathbf{x}} \mathrm{F}(\tilde{\mathbf{x}})$ is positive definite, then $\tilde{\mathbf{x}}$ is a local minimum, if $H_{\mathbf{x}} \mathrm{F}(\tilde{\mathbf{x}})$ is negative definite, then $\tilde{\mathbf{x}}$ is a local maximum, and if $H_{\mathbf{x}} \mathrm{F}(\tilde{\mathbf{x}})$ has eigenvalues with mixed signs, then $\tilde{\mathbf{x}}$ is a saddle-point.

In constrained optimization, which is our case, the Hessian test is extended to the Bordered Hessian test to account for the constraints - the Hessian has to be positive/negative definite at $\tilde{\mathbf{x}}$, while the gradient of the constraints has to be zero (at $\tilde{\mathbf{x}}$ ), for $\tilde{\mathbf{x}}$ to be a minimum/maximum [13]. Given an $n$ dimensional functional $\mathrm{P}(\mathbf{x})$ to be maximized, subject to a set of $m$ constraints $\mathbf{g}(\mathbf{x})$, the Bordered Hessian of the corresponding Lagrangian functional $\mathrm{F}(\mathbf{x}, \boldsymbol{\lambda})=\mathrm{P}(\mathbf{x})+$ $\boldsymbol{\lambda}^{T} \mathbf{g}(\mathbf{x})$, is defined as:

$$
\bar{H} \mathrm{~F}(\mathbf{x}, \boldsymbol{\lambda})=\left[\begin{array}{cc}
0_{m \times m} & \nabla \mathbf{g}(\mathbf{x})_{m \times n} \\
\nabla \mathbf{g}(\mathbf{x})_{n \times m}^{T} & H_{\mathbf{x}} \mathrm{F}(\mathbf{x}, \boldsymbol{\lambda})_{n \times n}
\end{array}\right]_{(m+n) \times(m+n)},
$$

where the Hessian of the Lagrangian functional is bordered by the Jacobian of the constraints $\nabla \mathbf{g}(\mathbf{x})$, and padded by a corner-block of zeros. In our case $n=3$ and $m=1$, therefore, the Bordered Hessian is a $4 \times 4$ matrix. The Bordered Hessian is rank deficient and cannot satisfy the definiteness conditions of the Hessian test. However, an extremum, $\tilde{\mathbf{X}}=(\tilde{\mathbf{x}}, \tilde{\boldsymbol{\lambda}})$, of the constrained optimization can be categorized using the following alternating sign tests:

$$
\begin{gathered}
(-1)^{m}\left|\bar{H}_{r} \mathrm{~F}(\tilde{\mathbf{X}})\right|>0 \text { strict minimum, } \\
(-1)^{r}\left|\bar{H}_{r} \mathrm{~F}(\tilde{\mathbf{X}})\right|>0 \text { strict maximum, } \\
r=m+1, \ldots, n, \\
\text { where : } \bar{H}_{r} \mathrm{~F}=\left[\begin{array}{cc}
0_{m \times m} & \nabla \mathbf{g}_{m \times r} \\
\nabla \mathbf{g}_{r \times m}^{T} & H_{\mathbf{x}} \mathrm{F}_{r \times r}
\end{array}\right]_{(m+r) \times(m+r)},
\end{gathered}
$$

where $\nabla \mathbf{g}_{m \times r}$ denotes the first $r$ columns of the Jacobian $\nabla \mathbf{g}(\mathbf{x})$ and $H_{\mathbf{x}} \mathrm{F}_{r \times r}$ denotes the principal $r \times r$ sub-matrix of the Hessian $H_{\mathbf{x}} \mathrm{F}_{n \times n}$. There are other ways of categorizing the extremum $\tilde{\mathbf{X}}$ into a maximum, or a minimum, e.g. using differential geometry as proposed in [7].

Tractography The continuous version of Streamline tractography [5] defined for DTI, considers a fiber tract as a 3D space curve parametrized by its arclength, $\mathbf{r}(s)$, and describes it by its Frenet equation:

$$
\frac{d \mathbf{r}(s)}{d s}=\mathbf{t}(s)=\epsilon_{1}(\mathbf{r}(s)), \quad \mathbf{r}(0)=\mathbf{r}_{0}
$$


where $\mathbf{t}(s)$ the tangent vector to $\mathbf{r}(s)$ at $s$ is equal to the unit major eigenvector $\epsilon_{1}(\mathbf{r}(s))$ of the diffusion tensor at $\mathbf{r}(s)$. This implies that fiber tracts are locally tangent to the major eigenvector of the diffusion tensor.

Integrating Eq.8 requires two things - first, a spatially continuous tensor (or SDF) field, and second, a numerical integration scheme. [5] proposed two approaches for estimating a spatially continuous tensor field from a discrete DTI tensor field, namely approximation and interpolation. They also proposed the Euler's method, the 2nd order Runge-Kutta method, and the adaptive 4th order Runge Kutta method as numerical integration schemes. Finally, for stopping they proposed four criteria - the tracts are within the image volume, the tracts are in regions with FA value higher than a threshold, the curvature of a tract is smaller than a threshold, and that a tract is better aligned with the major eigenvector in the next spatial location than any of the two other eigenvectors.

We adapt this tractography algorithm to SDFs with multiple maxima by modifying Eq.8 to:

$$
\frac{d \mathbf{r}(s)}{d s}=\eta_{\theta_{\min }}(\mathbf{r}(s)), \quad \mathbf{r}(0)=\eta_{\max }(0)
$$

where $\eta_{i}(\mathbf{r}(s))$ are all the unit maxima vectors of the SDF extracted by our method at $\mathbf{r}(s), \eta_{\max }$ is the unit maximum vector whose function value is the largest amongst all the $\eta_{i}$, and $\eta_{\theta_{\text {min }}}$ is the unit maximum vector in the current step that is most collinear to the unit maximum vector followed by the integration in the previous step. Eq.9 and the initial condition state that at the starting point we begin integrating along the dominant maximum direction, and at each consecutive step we first extract all the maxima of the SDFs and choose the maximum direction most collinear to the maximum direction from the previous integration step, to move forward in the integration.

Since we require a continuous field of SDFs for integrating Eq.9, we consider the Euclidean interpolation of the SDFs, which is equivalent to the L2-norm interpolation of the SDFs, since the SHs form an orthonormal basis [12]. For the numerical integration scheme we employ the 2nd order Runge-Kutta method due to its robustness and simplicity with an integration step of length $0.5 \mathrm{~mm}$.

For stopping we only use two criteria - the tracts are within the image volume, and tracts aren't allowed to have high curvatures, or the radius of curvature of tracts should be smaller than $0.87 \mathrm{~mm}$. Currently we don't employ any stopping criteria based on anisotropy indices because indices such as GFA for complex SDFs show similar contrasts to FA, and therefore have low values in regions with fiber crossings.

Since Streamline tractography traces fibers that are always tangent to the maxima of the local SDF, these fibers can be unnaturally "wriggly". Due to acquisition noise the estimated discrete SDF field is generally spatially irregular. Thus closely following the local maximum can cause the fiber to wriggle. This effect can be important enough to violate the curvature criterion and cause the tracking algorithm to abort. Furthermore, partial voluming effects can also cause certain SDFs to lack maxima along the fiber direction, especially in voxels with fiber crossings, even when neighbouring SDFs may have maxima well aligned 
with the fiber direction. This can cause the tracking algorithm to suddenly deviate to another fiber track, violating again the curvature criterion.

DTI Tensorline tractography was proposed to specifically address these issues [21]. $\epsilon_{1}$ in Eq.8 was replaced by:

$$
\mathbf{v}_{\text {out }}=f \epsilon_{1}+(1-f)\left((1-g) \mathbf{v}_{\text {in }}+g \mathbf{D} \cdot \mathbf{v}_{\text {in }}\right),
$$

where $f, g$ are user defined weights, $\mathbf{v}_{\text {in }}, \mathbf{v}_{\text {out }}$ are the incoming and outgoing tangents respectively, $\mathbf{D}$ is the local diffusion tensor with $\epsilon_{1}$ its unit major eigenvector, and $\mathbf{D} \cdot \mathbf{v}_{\text {in }}$ is the tensor deflection (TEND) term. This smooths away unnatural kinks, and also helps to plough through regions with uncertainty, where $\mathbf{D}$ is oblate or spherical.

With general SDFs, D doesn't exist, but the SDFs have more complex geometries that are meant to better resolve the angular uncertainties in regions with crossings, implying that the TEND term can be ignored. Therefore, we adapt the Tensorline tractography to general SDFs with multiple maxima by replacing $\eta_{\theta_{\min }}$ in Eq.9 by:

$$
\mathbf{v}_{\text {out }}=f \eta_{\theta_{\text {min }}}+(1-f) \mathbf{v}_{\text {in }},
$$

with $f$ a user defined weight, and with $\mathbf{v}_{\text {in }}, \mathbf{v}_{\text {out }}$ as defined above. $\mathbf{v}_{\text {in }}$ acts like an inertial factor to maintain the fiber's general trend, and Eq.11 smooths away kinks and helps to navigate regions with SDFs affected by partial voluming.

Thus we have proposed a polynomial based maxima extraction method to correctly extract all the maxima of any SDF described non-parametrically in the $\mathrm{SH}$ basis. And we have extended the deterministic Streamline and Tensorline tractography methods to deal with the multiple maxima discerned by complex SDFs in regions with fiber crossings, to be able to track fibers more accurately through such regions.

\section{Experiments}

We illustrate our approach on ODF-SAs which are the orientation marginal distribution of the diffusion probability density function $\Psi(\theta, \phi)=\int_{0}^{\infty} P(r, \theta, \phi) r^{2} d r$ [1]. They are SDFs with multiple peaks aligned along directions with high probability of diffusion of water molecules, i.e. fiber directions, and can discern crossing fiber configurations. ODF-SAs are a good example of non-parametrically represented SDFs described in the real and symmetric SH basis. From the Diffusion Weighted Images (DWIs), we estimated rank-4 ODF-SAs and then tracked fibers from specific seed regions using our proposed maxima extraction method and extensions of deterministic Streamline and Tensorline tractographies. We experimented on a synthetic dataset, and on an in vivo human cerebral dataset.

Synthetic Dataset: To conduct controlled experiments with known ground truths, we used a multi-tensor approach parametrized by a diagonal tensor $\mathbf{D}=$ $[1700,300,300] \times 10^{-6} \mathrm{~mm}^{2} / \mathrm{s}$ to generate synthetic DWIs [12]. The DWIs were generated along 81 directions for a $b$-value of $3000 \mathrm{~s} / \mathrm{mm}^{2}$. The synthetic data 
was used to first validate our maxima extraction method with voxels with 2 , and 3 fibers crossing. It was then used to generate a synthetic image of overlapping fiber bundles to validate fiber tracking using DTI tracking and ODF-SA tracking.

In vivo Human Cerebral Dataset: The in vivo human cerebral data was acquired with 60 encoding gradient directions, a $b$-value of $1000 \mathrm{~s} / \mathrm{mm}^{2}$, twicerefocused spin-echo $\mathrm{EPI}$ sequence, with $\mathrm{TE}=100 \mathrm{~ms}$, and $\mathrm{TR}=12 \mathrm{~ms}$. It has isotropic voxels of $1.7 \mathrm{~mm} \times 1.7 \mathrm{~mm} \times 1.7 \mathrm{~mm}$, with three repetitions, and was corrected for subject motion. It was acquired on a whole-body $3 \mathrm{~T}$ Siemens Trio scanner [3]. This dataset was used to compare DTI tractography and ODFSA tractography. We tracked specifically from seeds with the Corpus Callosum (CC). It is well known that due to the important crossings between the lateral radiations of the $\mathrm{CC}$ and Pyramidal Tract (PT) it is difficult to track the lateral radiations of the CC. We used the ODF-SA and our modified tracking algorithms to specifically recover these lateral radiations of the CC [19]. We also tracked the PT and the Superior Longitudinal Fasciculus (SLF) which crosses the lateral radiations of the $\mathrm{CC}$ and the $\mathrm{PT}$ to validate our tracking method in regions where all 3 fiber bundles cross.

\section{Results}

Figure-1 shows the results on the synthetic multitensor data test. In Fig-1a and Fig-1b we present the results of our maxima extraction. These voxels represent 2 and 3 fiber-crossings. All the extrema were extracted and categorized: maxima in thick yellow, minima in green, and saddle-points in thin blue. In the case of the 2 fiber configuration, even the unimportant maxima along the $z$-axis was correctly detected.

Figure-1c,d,e, present a synthetic dataset image with 2 fiber bundles crossing. Fig-1c shows the estimated ODF-SAs with the seed region highlighted in grey in the background. Fig-1d is the result of DTI Tensorline tracking with $f=0.3$ and $g=0$ (Eq.10). These weights imply that the TEND term was ignored and only the inertia term $\mathbf{v}_{\text {in }}$ played a role. These weights were chosen to make the DTI Tensorline tracking comparable to the ODF-SA Tensorline tracking. The ODF-SA Tensorline tracking with $f=0.3$ (Eq.11) is visible in Fig-1e. A closer inspection at the junction areas clearly indicate that DTI tracking has no crossings, and the fibers bend to accommodate for partial voluming, whereas in ODF-SA tracking the fibers cross each other as expected.

We also compared the computation time of our maxima extraction method to that of the discrete mesh search method proposed in [12], where a 16th order icosahedral tessellation of the sphere with 1281 samples of the SDF on a hemisphere was used. Comparing neighbouring directions in this tessellation revealed an angular resolution limit of $\sim 4^{\circ}$. Our maxima extraction can be considered as the limiting case of the discrete mesh search approach. Choosing the ODFSA Tensorline algorithm and only changing the maxima extraction method, we found the computation times to be highly comparable. Our method clocked 17 mins 34 secs while the discrete mesh search registered 15mins 45 secs. 
Results of the in vivo human cerebral dataset are presented in Figure-2. Neither the estimated DTI field nor the ODF-SA field were regularized prior to tracking. Tracking was seeded in regions of the CC. It is well known that fibers radiating laterally from the CC cross the PT [19], and are hard to track. This is clearly seen in Fig-2a which presents the DTI Streamline tractography result. This is improved by using DTI Tensorline tractography (parameters as above), as seen in Fig-2b. However, only a select few lateral radiations are found. A much richer distribution of fibers from the CC are tracked using the ODF-SA Tensorline tractography (parameters as above), as seen in Fig-2c. Fig-2d highlights ODF-SA Tensorline tractography by presenting fibers from three bundles that are known to cross. In red is the CC (left-right), in blue is the PT (superioinferior), and in green is the SLF (anterio-posterior). The close up in Fig-2e clearly shows fibers traced through a region where all three bundles cross.

\section{Conclusion}

In this paper, we first proposed a polynomial based approach for correctly extracting the maxima of any non-parametrically represented SDF in the SH basis. Recently a number of such HARDI SDFs have been proposed to infer accurately the micro-architecture of the underlying cerebral tissue. We illustrated our method on the ODF-SA. Our approach guarantees to analytically bracket all the maxima, and to numerically refine them to any precision, to accurately quantify the SDF's geometry. It can be seen as the limiting case of a discrete mesh search, with the complete mathematical framework of optimization. However, it differs from optimization since it's not a local search and isn't dependent on an initial solution. Further, all the maxima are found together.

Maxima extraction is sensitive to both the estimation of the SDFs, which can be affected by signal noise, and the localization of the SDFs' maxima after estimation, which can be affected by the maxima extraction process. Our method is independent of the SDFs' estimation process - it computes on the SDFs' SH coefficients after these have been estimated from the signal and provides an error free localization of the correct maxima. However, when the SDFs estimation isn't robust, the maxima can be affected by signal noise.

As an application of our maxima extraction method, we also proposed an extension of the DTI Streamline and Tensorline algorithms. We tested on synthetic and in vivo human cerebral datasets. Using the ODF-SA Tensorline algorithms, we showed that we were able to dramatically improve deterministic tractography results. We were able to recover a rich distribution of the lateral radiations of the CC [19], which are hard to track since they cross the PT. Further, by tracking from seeds in the CC, PT, and SLF, we were able to correctly trace through regions where all three fiber bundles cross.

\section{References}

1. Aganj, I., Lenglet, C., Sapiro, G., Yacoub, E., Ugurbil, K., Harel, N.: Reconstruction of the orientation distribution function in single and multiple shell Q-ball 

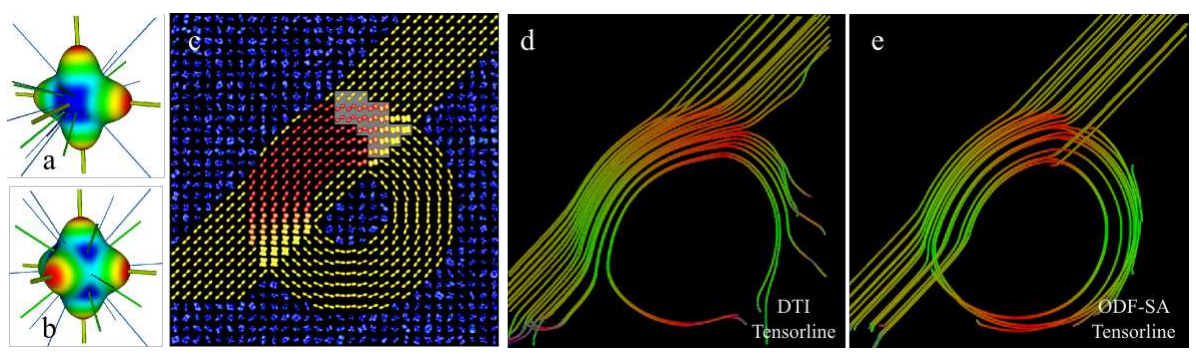

Fig. 1. (a,b): Extrema extraction \& Categorization on synthetic data. Glyph-colour: red: high anisotropy, blue: low anisotropy Lines: thick-yellow: Maxima, thin-blue: Saddle points, green: Minima. a) 2-fibers b) 3-fibers. (c,d,e) Fiber tracking on synthetic dataset image with 2 fiber bundles crossing. c) ODF-SA glyphs with seeds highlighted as grey in the background. d) DTI Tensorline tracking. e) ODF Tensorline tracking. DTI tracking never shows crossings, whereas ODF-SA tracking clearly shows crossings.

imaging within constant solid angle. Magnetic Resonance in Medicine 64(2), 554566 (2010)

2. Alexander, D.C.: Maximum Entropy Spherical Deconvolution for Diffusion MRI. In: Image Processing in Medical Imaging. pp. 76-87 (2005)

3. Anwander, A., Tittgemeyer, M., von Cramon, D.Y., Friederici, A.D., Knosche, T.R.: Connectivity-based parcellation of broca's area. Cerebral Cortex 17(4), 816825 (2007)

4. Basser, P.J., Mattiello, J., LeBihan, D.: MR diffusion tensor spectroscopy and imaging. Biophysical Journal 66(1), 259-267 (1994)

5. Basser, P.J., Pajevic, S., Pierpaoli, C., Duda, J., Aldroubi, A.: In vivo fiber tractography using DT-MRI data. Magnetic Resonance in Medicine 44(4), 625-632 (October 2000), http://view.ncbi.nlm.nih.gov/pubmed/11025519

6. Behrens, T.E.J., Johansen-Berg, H., Jbabdi, S., Rushworth, M.F.S., Woolrich, M.W.: Probabilistic diffusion tractography with multiple fibre orientations. what can we gain? NeuroImage 34(1), 144-155 (2007)

7. Bloy, L., Verma, R.: On computing the underlying fiber directions from the diffusion orientation distribution function. In: MICCAI '08: Proceedings of the 11th international conference on Medical Image Computing and Computer-Assisted Intervention - Part I. pp. 1-8. Springer-Verlag, Berlin, Heidelberg (2008)

8. Catani, M., Howard, R.J., Pajevic, S., Jones, D.K.: Virtual in vivo interactive dissection of white matter fasciculi in the human brain. NeuroImage 17, 77-94 (2002)

9. Ciccarelli, O., Catani, M., Johansen-Berg, H., Clark, C., Thompson, A.: Diffusionbased tractography in neurological disorders: concepts, applications, and future developments. Lancet Neurol 7(8), 715-27 (2008)

10. Descoteaux, M., Angelino, E., Fitzgibbons, S., Deriche, R.: Apparent diffusion coefficients from high angular resolution diffusion imaging: Estimation and applications. Magnetic Resonance in Medicine 56, 395-410 (2006)

11. Descoteaux, M., Angelino, E., Fitzgibbons, S., R.Deriche: A Fast and Robust ODF Estimation Algorithm in Q-Ball Imaging. In: Third IEEE ISBI: from Nano to Macro. pp. 81-84. Arlington, Virginia, USA (Apr 2006) 


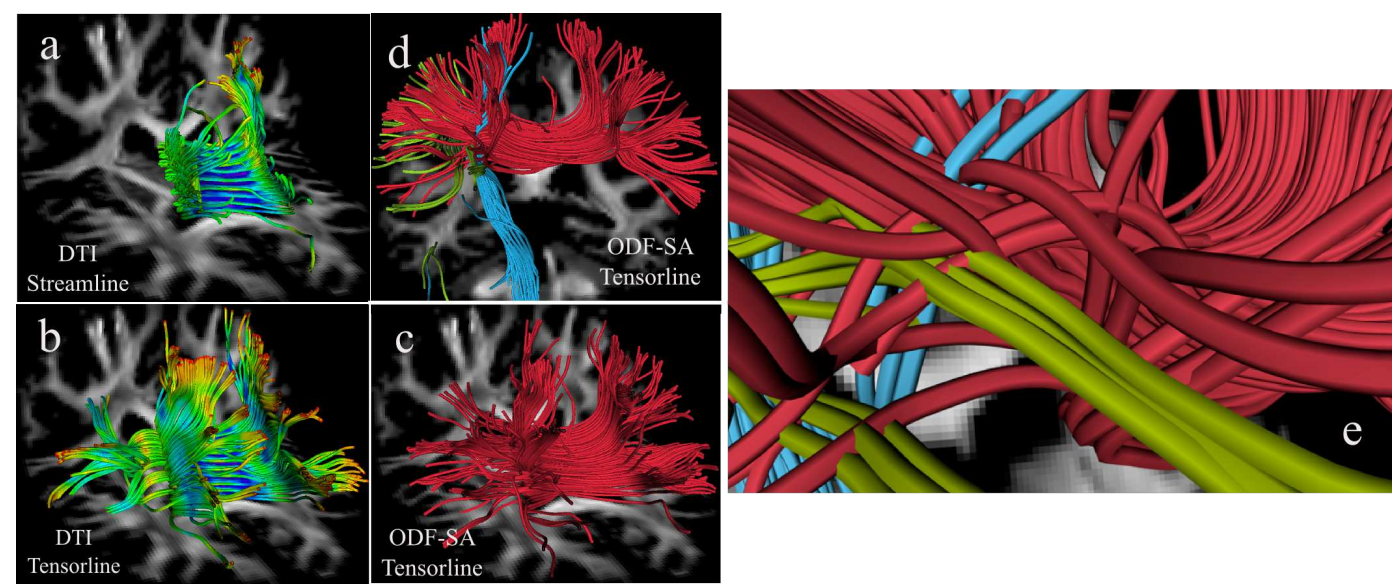

Fig. 2. Tractography from the Corpus Callosum (CC). a) DTI Streamline cannot recover lateral radiations of CC. b) DTI Tensorline improves on (a), some lateral radiations are recovered. c) ODF-SA Tensorline recovers a rich distribution of the CC. d) ODF-SA Tensorline tracking from seeds in the CC (red: left-right), Pyramidal Tract (PT: superio-inferior: blue) and Superior Longitudinal Fasciculus (SLF: anterioposterior: green). e) Zoom: Tracking visible through regions where all 3 fiber bundles cross.

12. Descoteaux, M., Deriche, R., Knosche, T.R., Anwander, A.: Deterministic and Probabilistic Tractography Based on Complex Fibre Orientation Distributions. IEEE Transactions on Medical Imaging 28(2), 269-286 (2009)

13. Im, E.: Hessian sufficiency for bordered Hessian. Res. Lett. Inf. Math. Sci. 8, 189196 (2005)

14. Jeurissen, B., Leemans, A., Jones, D.K., Tournier, J.D., Sijbers, J.: Probabilistic fiber tracking using the residual bootstrap with constrained spherical deconvolution. Human Brain Mapping (2010)

15. Bernard Mourrain, Jean-Pascal Pavone: Subdivision methods for solving polynomial equations. Journal of Symbolic Computation 44, 292-306 (2009)

16. Ozarslan, E., Shepherd, T., Vemuri, B., Blackband, S., Mareci, T.: Resolution of complex tissue microarchitecture using the diffusion orientation transform (dot). NeuroImage 31(3), 1086-1103 (2006)

17. Tournier, J.D., Calamante, F., Gadian, D., Connelly, A.: Direct estimation of the fiber orientation density function from diffusion-weighted MRI data using spherical deconvolution. NeuroImage 23, 1176-1185 (2004)

18. Tuch, D.: Q-ball imaging. Magnetic Resonance in Medicine 52(6), 1358-1372 (2004)

19. Türe, U., Yasargil, M., Friedman, A., Al-Mefty, O.: Fiber dissection technique: lateral aspect of the brain. Neurosurgery 47(2), 417 (2000)

20. Wedeen, V.J., Hagmann, P., Tseng, W.Y.I., Reese, T.G., Weisskoff, R.M.: Mapping complex tissue architecture with diffusion spectrum magnetic resonance imaging. Magn. Reson. Med. 54(6), 1377-1386 (2005)

21. Weinstein, D.M., Kindlmann, G.L., Lundberg, E.C.: Tensorlines: Advectiondiffusion based propagation through diffusion tensor fields. Visualization Conference, IEEE 0, 40 (1999) 